\title{
Coming to terms with heritability
}

\author{
Scott F. Stoltenberg \\ Department of Psychology, University of Illinois at Urbana-Champaign, 603 East Daniel Street, Champaign, IL \\ 61820, USA; Current address: University of Michigan, Alcohol Research Center, Department of Psychiatry, 400 \\ E. Eisenhower Parkway, Suite 2A, Ann Arbor, MI 48108, USA
}

Received 17 May 1996 Accepted 2 August 1996

Key words: heritability, heritable, heredity, hereditary, selectability

\begin{abstract}
The complex mechanisms of heredity are little appreciated by non-specialists, in some measure, because of misunderstandings that are perpetuated when words used for technical terms have other, more widely understood, folk meanings. When a word has both technical and folk meanings, it is the responsibility of the specialist to avoid promoting confusion by either using extremely cautious and precise language when using the term or, in cases when confusion is inevitable, abandoning the term in favor of one without a widely understood folk meaning. The study of heredity is beset by such confusion, and the term heritability appears to be at the heart of some of the confusion. In this article, I discuss both the technical and folk meanings of heritability and examine the bridge between them. By continuing to use the term heritability, we risk promulgating serious misunderstanding about the workings of heredity, therefore I suggest selectability as an alternative term to avoid such pitfalls.
\end{abstract}

\section{Introduction}

It is curious that, although the fact of heredity is familiar to everybody from everyday observation, heredity is one of the most widely and persistently misunderstood phenomena of nature. The main source of the misunderstanding is an ambiguity of our language... (Dobzhansky, 1964, p. 7)

Despite unprecedented progress in the scientific understanding of heredity in the years since Dobzhansky penned this eloquent statement, no real progress has been made toward clearing up the misunderstandings that are a direct result of the ambiguities to which he referred. Though scientists are largely responsible for such misunderstandings - because they have taken words with widely understood folk meanings and used them to describe precise mechanisms and quantities that are inconsistent with the more well-known meanings of those words - no serious attempt has been made by them to reduce such ambiguity in our language regarding heredity. On the contrary, terms such as 'heritability' and its derivative 'heritable' - which perpetuate a basic misunderstanding of the mechanisms of heredity - have become commonplace in the scientific literature.

Many of the technical terms used by professional geneticists have meanings in the English language other than those they intend, and those non-technical meanings are rather old and widely understood, whereas the technical meanings are rather new and are understood only by specialists. One of the fundamental aspects of heredity that is rarely fully understood by non-specialists has to do with what it is that is actually transmitted from parents to offspring. In a genetic sense, only the constituents of the gametes are inherited, traits are developed anew in each individual. However, when scientists make statements suggesting that a behavior is heritable they perpetuate the misunderstanding that behaviors are passed down from parents to offspring much like a baton that is passed from one relay runner to the next. The common or folk understanding of heredity is closely tied to very old definitions which have more to do with handing down property and/or titles from one generation to the next than they do with genetics. 
To better understand the basis of the confusion between folk and technical definitions I examine, in this article, some terms associated with heredity, but my main focus is on the term heritability because of its importance in both technical and popular writings. My purpose is to present an examination of the ways that the word heritability has been used in the English language in some detail and to discuss some of the confusions and misunderstandings that have arisen as a result of heritability's dual meaning. First, I discuss the technical meaning of heritability, including its two definitions, both narrow and broad. Second, I discuss some of the common, or folk, meanings of terms associated with heredity, including heritability. Third, I address the question 'What is inherited?' to explore the differences between what the specialists and the non-specialists understand about heredity. Finally, I suggest strategies aimed at reducing the confusion fostered by the contamination of the technical usage by the folk usage, including a suggestion for an alternative technical term.

In this article, I do not discuss in detail the pros and cons of making heritability estimates for traits in human populations. I leave that argument, based on statistical concerns and on certain necessary - often violated - assumptions, to others more qualified than I. My main task is to explore some of the issues surrounding the ambiguity of our language with respect to heredity.

\section{Technical heritability}

Heritability estimates - variance ratios which can range from 0.0 to 1.0 - were first used by breeders to predict the effectiveness of artificial selection for particular trait expressions in a given population under carefully specified living conditions (e.g., Lush, 1937). For economically important traits, such as milk production in cows, it can be important to have some idea of whether a lengthy (therefore costly) program of selective breeding is likely to result in the desired selection gains. For a given population under carefully specified conditions, predicting the response to selection for the expression of various traits can be rather accurately done in the early generations of selection; however, predicting the response in later generations often becomes quite difficult (Lerner, 1958). Because an estimate of heritability is a property of a population at a specific time and in a specific environment, changes in the population's gene pool (e.g., as a result of selection), or in the envi- ronment, can change heritability estimates. Whereas heritability estimates can provide some useful information for breeders, it has been long recognized that they are but a small piece in an exceedingly complex puzzle (Lush, 1937; Lerner, 1958).

The technical term heritability refers to a specific statistic for estimating the proportion of trait variance that is accounted for (in a statistical sense) by genetic factors (but see Lewontin, 1974). However, the language that surrounds the partitioning of variance is prone to misunderstanding in its own right (Lewontin, 1974; Kempthorne, 1978), therefore I avoid using terms such as 'due to' or 'caused by' when referring to the statistical relations between an independent variable and a dependent variable (e.g., in an analysis of variance [ANOVA]), but instead use terms such as 'associated with' to avoid deterministic implications.

Of course, animal breeders were not alone in their interest in heritability estimates. In the first part of the century, theoretically oriented quantitative geneticists were in the early stages of developing their science and some found that heritability estimates filled an important niche in their models of the relation between heredity and quantitative traits. Wright was especially important in the development of the ideas surrounding heritability estimates; in fact, he made use of the concept in his early work on coat color in guinea pigs (Wright, 1920), although it was not explicitly named until 1940 by Lush (Lush, 1940; see Bell, 1977). Other quantitative geneticists, however, were not enamored by heritability estimation. In fact, Fisher once described it as 'one of those unfortunate short-cuts which have emerged from biometry for lack of a more thorough analysis of the data.' (Fisher, 1951, cited in Wahlsten, 1994b).

Since the birth of the discipline of behavior-genetic analysis in the late 1950s, researchers have often applied the statistical approaches developed by animal breeders and quantitative geneticists, specifically heritability estimation, in efforts to understand the relations between heredity and perhaps the most complex phenotype, behavior. In an early review of the field of behavior-genetic analysis, McClearn (1962) states that 'one of the central concepts of genetics is that of heritability' (p. 234, his emphasis). Curiously, McClearn (1962) did not frequently use the term heritability; however, he did refer to the concept fairly regularly: 'weighing the relative effects of nature and nurture' (p. 196), 'relative contributions of heredity and environment' (p. 206), and 'relative influences of genetic differences and environmental differences 
in determining the individual variability in phenotypes'. (p. 210). Clearly, by the early 1960s heritability estimates had gained a strong foothold in the study of heredity-behavior relations; however, not all behaviorgeneticists agreed that they were a 'central concept' of the field (e.g., Hirsch, 1967, p. 423).

Heritability estimates are now firmly entrenched in the field of behavior genetics, although there are differing opinions regarding their utility in research with human subjects (e.g., McGuire \& Hirsch, 1977; Kempthorne, 1978; Plomin et al., 1990; Tesser, 1993; Wahlsten, 1994a). In behavior-genetic analysis heritability estimates are one of the most frequently calculated statistics when humans are the research subjects, whereas they are less frequently calculated when animals are. Of the 98 articles published in volumes 24 and 25 (1994 \& 1995) in the journal Behavior Genetics (excluding abstracts, book reviews, etc.) $33 \%$ (32) reported heritability estimates. Of those articles reporting heritability estimates, $87.5 \%$ (28) used human subjects, while the remaining $12.5 \%$ (4) used animal subjects. There is a clear bias in favor of calculating heritability estimates when the research subjects are human.

It is rather disconcerting that heritability estimation is more common in human than in animal behaviorgenetic analysis for two main reasons. First, the primary use of heritability estimates is to predict or to assess the results of selective breeding, which is obviously proscribed in human populations. Second, human populations are much more unlikely to satisfy the host of assumptions that undergird partitioning behavioral variance into the $\mathrm{P}=\mathrm{G}+\mathrm{E}$ model. Such assumptions include no dominance deviation, no epistasis, no assortative mating, no genotype $\times$ environment $(\mathrm{G} \times \mathrm{E})$ interaction, and no GE covariance (Falconer, 1989). Most or all of these assumptions are unlikely to be met in human populations (McGuire \& Hirsch, 1977).

Of course, it is a common practice to include such effects in the model being tested and drop those terms that fail to reach a predetermined significance criterion. In such a situation one needs to consider the statistical power of the comparison in question. Wahlsten's (1990) discussion of the power of the ANOVA model to detect heredity-environment interactions is a wake-up call for all researchers who routinely use ANOVAs to carefully consider power questions when designing and trying to interpret the results of experiments. Regardless of the model being tested, dropping terms that do not reach statistical significance does not change the biological system being studied.
J.L. Lush is generally credited with being the first to use the term heritability in a technical sense, although others - in particular, some plant breeders - had begun using it more loosely somewhat earlier (Bell, 1977). Building on the ideas of Johannsen, Wright and Fisher, Lush was the first to precisely define heritability as 'the fraction of the observed variance which was caused by differences in heredity' (Lush, 1940, p. 293). Recently, Plomin et al. (1994) defined heritability as 'the proportion of phenotypic variance in a population that can be attributed to genetic influences' (p. 1734). Clearly, the technical definition of heritability has remained essentially unchanged for more than half a century.

It is almost certain that Lush (1940, p. 295) coined the terms narrow and broad sense heritabilities: 'The difference between narrow and broad definitions ... consists in whether the epistatic and dominance deviations are considered'. Narrow heritabilities, on the one hand, are those of most interest to the selective breeder in that they measure the extent to which, in a given population in a specific environment, variance in the trait of interest is associated with additive genetic (i.e., allelic) variance - that which is transmissible from parents to offspring. Narrow heritabilities therefore provide a way to predict whether selective breeding for that trait in that population will be successful (Falconer, 1989). Broad heritabilities, on the other hand, estimate the extent to which variance in the trait of interest is associated with the genotype - including dominance deviation and interaction variance - for a given trait in a population raised in a specific environment. Broad heritability estimates are of little interest to the geneticist because they can only provide upper limits to estimates of transmissible genetic variance (i.e., narrow heritability). By including dominance deviation and interaction variance - which are frustratingly difficult to measure even in well controlled experiments - estimates of the trait variance due to transmissible genetic variance are biased to an unknown degree. Narrow and broad heritability estimates are the same only when dominance deviation and interaction variance are absent (Falconer, 1989). It is not uncommon for the assumptions of no dominance deviation and no interaction variance to be made, however, but is quite unlikely that such a simple (additive) relation exists between complex human behaviors and heredity (e.g., Wahlsten, 1994a).

While it has long been known that statistical interactions can be eliminated by scale transformations (e.g., Lerner, 1958; Falconer, 1989), such manipulations do not alter the nature of biological interactions that occur throughout ontogeny, but can obscure 
perhaps the most important and interesting aspects of development. The complexities inherent in development are becoming better understood and nowhere else can this progress be better seen than in the fruit fly (see Lawrence, 1992 for a review), however, much still remains to be learned. When such in depth (experimental) studies are performed, the non-linear nature of development is revealed. Simplistic, linear models do not do justice to the wondrous complexity of ontogeny.

The distinction between narrow (h2) and broad (H2) heritability estimates is crucial, but is often ignored or unappreciated by psychologists (McGuire $\&$ Hirsch, 1977). Narrow heritability estimates are key in predicting or assessing the effectiveness of artificial selection in that they provide a way to gauge the extent to which variance in transmissable genetic factors is related to phenotypic variance in a given population. Therefore, h2 estimates are of interest in terms of parent-offspring resemblance. Broad heritability estimates assess the extent to which genotypic variance is related to phenotypic variance in a given population. It is important to stress that $\mathrm{H} 2$ estimates include transmissable genetic variation in addition to variation that results from sources idiosyncratic to the genotypes present in the population and the environments to which the population is exposed. Intra- (dominance) and inter-(epistasis) locular interactions and heredityenvironment interactions are all included as genotypic variance in broad heritability estimates. Narrow and broad heritability estimates are very different creatures and are by no means interchangeable.

The two approaches that are most commonly used to make heritability estimates for traits in human populations are twin and adoption studies (Plomin et al., 1990). In general, with twin studies one can estimate $\mathrm{H} 2$, whereas with adoption studies it is possible to estimate h2 (Falconer, 1989). It is clearly inappropriate to compare h2 with $\mathrm{H} 2$ directly, because they estimate different quantities, however, such comparisons are not uncommon. In fact, it is sometimes difficult to determine whether the author is referring to $\mathrm{h} 2$ or $\mathrm{H} 2$ because both estimates are referred to as heritability and are often represented by h2. For example, Emde et al. (1992) estimated 'heritability (h2)', by 'doubling the between MZ (monozygotic) and DZ (dizygotic) correlations' (p. 1445). Because this method is used to calculate broad heritability (Plomin et al., 1990, p. 320-322), it is inappropriate to label the quantity h2. When h2 and H2 estimates are referred to interchangeably it becomes difficult to critically assess the analyses and subsequent discussion presented.
Heritability estimates have been successfully used by animal and plant breeders to predict the response to artificial selection (Lerner, 1958), however, h2 estimates have more recently given way to multivariate statistical techniques that can incorporate more real-life biological complexity into the models (Arnold, 1994). It is ironic that heritability estimates are made most often in behavior-genetic research using human subjects, where the only real application of h2 estimates selective breeding - is prohibited.

\section{Folk heritability}

The word heritability has another distinct meaning in the English language, not wholly unrelated to the technical meaning, but in no way interchangeable with it. Heritability is defined as 'the quality of being heritable, or capable of being inherited' (OED2).

The Oxford English Dictionary (OED2) provides three references of some of the early uses of heritability that span the greater part of the 19th century. The first is in Fraser's Magazine (1832): 'This tax, thus securing the heritability of offices, was not perpetual'. The second is in the Proceedings of the American Academy of Arts (1882) by noted naturalist Asa Gray: 'The importance of heritability, which is an essential part of Darwinism, would seem to have had a significant illustration in the person of its great expounder'. The third is in Advance (1890): 'Did you ever think about the heritability of such qualities?' In each case, it is obvious that heritability is used as a synonym for 'inheritance'. Clearly, prior to the advent of the technical definition (ca. 1940), heritability was widely understood to mean 'capable of being inherited'. Used in this way, if some thing is to be inherited, that thing is to be passed from one to another. In a legal sense, such an interpretation is completely justifiable. However, in a genetic sense such an interpretation does not take into account the complexities of heredity and development which make the folk definition of heritability misleading when the technical definition of the word is intended.

\section{What is inherited?}

Perhaps the most misunderstood aspect of biological inheritance concerns what it is that is actually transmitted from parents to offspring. In the strictest genetic sense, it is inappropriate to speak of traits or characteristics being inherited; only the constituents of 
the gametes are passed from one generation to the next. The mechanisms of meiosis and development preclude the transmission of traits across generations; unique genotypes are formed at fertilization which then develop through interactions with idiosyncratic environments to form individuals. It is beyond question that for some traits offspring do resemble their parents and that those traits were influenced by the parents' genetic contribution. It is not appropriate, however, to say that those traits are inherited. Traits are not transmitted from parents to offspring, only the constituents of the gametes are, all else is development (see King, 1981).

Few non-specialists recognize the distinction between the technical and folk definitions of the terms surrounding heredity. Generally, folk definitions lack the precision necessary for good technical definitions and are therefore broader in scope. The two main folk definitions of the word 'inherit' should serve as excellent examples of the ways in which such definitions can differ from technical ones, while at the same time hinting at, albeit falling short of, the technical meaning.

First, perhaps the most common folk definition of inherit refers primarily to legal matters:

To take or receive (property, esp. real property, or a right, privilege, rank or title) as the heir of the former possessor (usually an ancestor) at his decease; to get, or come into possession of, by legal descent or succession (OED2).

Clearly, under this definition items are passed from one person to another, more or less intact (ignoring inheritance taxes for the sake of discussion). The eldest son was the usual recipient of such items. This particular meaning of inherit is quite old, being part of the English language since at least the 15 th century and is very weakly linked with the technical (biological) understanding of inherit, in that something is passed down from one generation to the next.

Second, the folk definition of inherit, that is perhaps the next most common, is closer to the technical (i.e., genetic) definition, however they are far from isomorphic.

To derive (a quality or character, physical or mental) from one's progenitors by natural descent; to derive or possess by transmission from parents or ancestry (OED2).

Many of us have heard it said that 'she has her mother's nose', or 'he got his bad temper from his father's side of the family' or something to that effect. Perhaps it is from this general understanding of 'natural descent' that our current technical understanding of heredity is derived. This particular definition of inherit has been a part of the English language since at least the 16th century, long before the mechanisms of heredity were understood in even a rudimentary way. Shakespeare (1601) used this form of inherit in All's Well That Ends Well I. ii. 22 'Youth, thou bear'st thy Fathers face, ..Thy Fathers morall parts Maist thou inherit too' (OED2).

It is important to note that in both of these folk definitions of inherit, those things that are passed from one person to another remain essentially intact. Whether one is referring to land or to a bad temper, to inherit is to receive something that is whole. This quality of passing down an item or a trait intact is an important distinction between the folk and the technical definitions of inherit.

To a geneticist, the only material that is inherited - transmitted from parents to offspring - consists of the constituents of the sperm cell from the father and of the egg cell (along with various proteins) from the mother. Only these cellular components are inherited in a strict genetic sense. The traits themselves are not passed down, but are developed anew in each individual as a result of a complex (idiosyncratic) series of interactions between biological material (e.g., genes, RNA, proteins) and the unique environment in which that individual is raised.

The cellular processes that result in sex cell formation, called gameteogenesis, are collectively known as meiosis. Understanding these processes is essential for a proper understanding of the relations between heredity and behavior because in them lie the key mechanisms of Mendelian genetics. However, psychologists have been slow to recognize the important role that meiosis plays in the generation of individual differences (Hirsch, 1963).

In the early stages of meiosis, homologous chromosomes pair up. While thus paired, homologues may exchange material, a process known as crossing over or recombination. Such recombination is the foundation for the multitude of linear mapping studies that have been and are currently being carried out by geneticists. More importantly, in the context of understanding 'What is inherited?', recombination is a crucial step in generating genotypic uniqueness. The newly produced (recombined) chromosomes are then packaged into gametes (sperm or egg) so that each gamete contains 23 chromosomes. Which of the two chromosomes from a given pair that is packaged into a sperm (or egg) is independent of that same process in any other pair, a process known as independent assortment. Recombi- 
nation and independent assortment, which have been appreciated only in this century, are so effective in producing diversity that it is statistically unlikely that two unrelated individuals will have the same genotype (Hirsch, 1963). More to the point, these mechanisms effectively shuffle genetic material so that a parent does not pass their genotype (entire genetic complement) to the next generation. Of course a parent does transmit genetic material to their offspring, but it is combined with genetic material from their mate to form unique genotypes at conception. By its nature, such biological inheritance is inconsistent with the folk notion of inheritance implied by sayings such as 'she has her mother's nose'.

\section{Heritable}

When a heritability coefficient for a trait (let's say trait $\mathrm{X}$ ) in a population is significantly different from zero, it is sometimes said that, 'trait X is heritable'. While such a transition is only a harmless 'short-hand' from one perspective, it is a reckless leap from another. In fact, it appears that this transition from non-zero heritability coefficients for a particular trait in a specific population to the statement that the trait is heritable is the crux of the confusion surrounding the use of the term heritability. The word heritable bridges the gap between a precisely defined technical term and a loosely defined, yet widely understood folk term. As described above, the folk and technical meanings of heritability are very different, but are easily confused. In addition, nonspecialists, unfamiliar with the technical meaning of heritability, understandably resort to the folk definition when confronted with the term. Use of the word heritable by specialists reinforces the interpretation of heritability in a folk sense because whereas heritable has a well-known folk definition, it has no technical definition.

I have been unable to locate a definition of heritable in the technical literature, though the term is used rather liberally. Often, in the introductory section of an article, the word heritable is used to discuss research findings, for example, 'alcoholism has been assumed to be highly heritable' and 'autism appears to be among the most heritable psychiatric disorders'. (Plomin et al., 1994; p. 1734). In this particular article, the word heritable was not defined, and in fact, its use preceded the technical term heritability (which was defined), a situation that is not uncommon. The reader can only assume that since heritable is undefined and precedes the technical definition of heritability, the writer intends the common or folk meaning of heritable, 'naturally transmissible or transmitted from parent to offspring; hereditary' (OED2). Such a definition is quite far from the technical definition of heritability in that it refers to the trait itself, not to variation in the expression of that trait in a population. This may appear to be a trivial difference, a quibble, but confusion over the distinction between the inheritance of traits in individuals and of variation in that trait in a population is a fundamental misunderstanding of the mechanisms of heredity and of the theoretical basis of heritability estimates.

It is not difficult to find the terms heritable or heritability in both scientific and popular publications. Recently, the American Psychologist published a 'Report of a Task Force Established by the American Psychological Association' entitled 'Intelligence: Knowns and Unknowns' (Neisser, et al., 1996) which was the 'authoritative report' on such issues inspired by the controversy that followed publication of The Bell Curve (Herrnstein \& Murray, 1994). The Task Force was chosen 'with the aim of representing a broad range of expertise and opinion' (p. 77). The resulting report can thus be considered the state of the art concerning intelligence. Unfortunately, with respect to associations between genetic factors and intelligence in humans, the art is rather underdeveloped. In the attempt to dispel one myth about heritability estimates, the report fosters another that is perhaps more damaging to understanding the nature of heredity:

A common error is to assume that because something is heritable it is necessarily unchangeable. This is wrong. Heritability does not imply immutability. As previously noted, heritable traits can depend on learning, and they may be subject to environmental effects as well (p. 86, my emphasis).

The point being made is a good one: significant heritability estimates for a trait in a population do not mean that the trait cannot respond to environmental manipulation. One common misunderstanding is that whenever a trait is said to be 'genetic' that it is hopelessly fixed; a notion that is clearly wrong. However, by using the term heritable - which is undefined technically, but has a widely understood folk definition (capable of being inherited, hereditary) - the authors of this report are perpetuating the misunderstanding that traits are inherited.

It is particularly noteworthy that many of those who specialize in the study of heredity-behavior relations 
also promulgate the mistaken notion that behaviors are inherited. McClearn's (1962) oft cited review of the history of behavior genetic analysis, before its coming to age as a discrete area of study, is titled 'The inheritance of behavior' (my emphasis). The masthead of Behavior Genetics, the journal of the Behavior Genetics Association, proclaims it to be: 'An international journal devoted to research in the inheritance of behavior in animals and man' (my emphasis). When such a misunderstanding of the fundamental mechanisms of genetics is advanced by the scientific establishment, it is little wonder that it is widespread in the popular literature.

\section{Selectability}

Apparently it does little good to warn against over simplifying the idea of heritability. Some feel it necessary to repeat those warnings each time they write. Others ignore the warnings. Much of the bulk of that which has been written in recent years about heritability in man comes from this.... Perhaps this is a basic argument for coining a new word ... (Lush, cited in Bell, 1977, p. 299, his emphasis).

To this point, I have outlined one of the main problems with the use of the term heritability by specialists: the folk meaning of the word is generally understood when the technical meaning is intended. The folk meaning of heritability has a much longer history in the English language than does its technical meaning and is much more widely used. The confusion between the folk and technical meanings of heritability is further fostered by the transition made by researchers from statistically significant, non-zero heritability coefficients for a trait in a population to claiming the trait itself to be heritable. The term heritable has no generally accepted technical definition, though it does have a well understood folk meaning (i.e., hereditary). Thus, the ambiguity in the English language surrounding the term heritability has seriously compromised its utility as a meaningful genetic statistic. What can and should be done to clear up such confusion?

There are two courses of action that I suggest; I am sure that, in addition to these, there are still others. Neither of these approaches is above criticism, in fact, any solution will assuredly have both proponents and opponents. It is my intention to stimulate thought and discussion about these matters, not to provide a final word on the matter in these pages.
A step that would be, at the same time, relatively painless and rather effective in reducing some of the confusion surrounding heritability is for researchers to avoid using the 'short-hand' whereby traits with statistically significant heritability coefficients are referred to as being heritable. By labeling traits as heritable, the bridge between technical heritability estimates and folk heritability is constructed. Since no explicit technical definition of heritable exists it is inevitable that the term is interpreted using its folk definition. The folk definition of heritable is not meaningful in the context of our current understanding of heredity. Such use is confusing at best and leads to widespread misunderstanding about the nature of heredity at worst. By refraining from using such short-hand researchers can at least stop confusing those less sophisticated than themselves.

Another approach to reducing some of the confusion surrounding heritability is to follow the advice of Lush and 'coin a new word' (cited in Bell, 1977). Such a word should present the idea 'precisely and in a way in which it cannot be misunderstood.' This solution is much more ambitious than the first. However, it has the potential to eliminate the confusion between the technical and folk meanings of heritability and also to reflect the theoretical underpinnings of the statistic.

The word 'selectability' may prove to be a satisfactory replacement for heritability. Selectability has no folk definition and thus cannot be subject to the same kind of confusion promoted by the use of heritability. The Oxford English Dictionary lists no matches for the word selectability. Of course, its root, select has been a part of the English language for centuries. Of select's several definitions, the one that most closely matches the intended technical meaning is: 'To choose or pick out something from a number; to make a selection'. In fact, Charles Darwin (1859) cites the word in a way that is consistent with the intended technical meaning: 'In man's methodical selection, a breeder selects for some definite object...'(OED2). Thus, in this case, the technical and folk meanings of the word are wholly similar, and in harmony with Lush's (1940) intentions for estimating such a statistic, that is, to provide an estimate of the expected results of artificial selection.

Admittedly, proposing to replace a term that has been in use for more than 50 years and has many proponents will almost assuredly be viewed in a negative light by some. Those researchers who routinely calculate heritability estimates for traits in human populations will certainly be the most difficult to persuade. To calculate a statistic called a 'selectability 
estimate' or to identify a trait in human populations as 'selectable' obviously carries baggage of its own, raising the specter of eugenics. Because of this, researchers would need to carefully consider the utility of calculating such a statistic, and when it is deemed necessary, careful use of language would be required. To begin to resolve some of the misunderstandings surrounding heredity, it is imperative that we come to terms with the ambiguity in our language and chart a course that avoids such pitfalls whenever possible.

\section{Acknowledgements}

Thanks to J. Hirsch and to F. Weizmann for critically reading an earlier version of the manuscript and to M.G. Terry for suggesting the term 'selectability'. This work was done with support from the University of Illinois Foundation.

\section{References}

Arnold, S.J., 1994. Multivariate inheritance and evolution: A review of concepts, in Quantitative Genetic Studies of Behavioral Evolution edited by C.R.B. Boake, University of Chicago Press, Chicago

Bell, A.E., 1977. Heritability in retrospect. J. Hered. 68: 297-300.

Emde, R.N., R. Plomin, J. Robinson, R. Corley, J.C. DeFries, D.W. Fulker, J.S. Reznick, J. Campos, J. Kagan \& C. Zahn-Waxler, 1992. Temperament, emotion and cognition at fourteen months: The MacArthur Longitudinal Twin Study. Child Dev. 63: 14371455.

Falconer, D.S., 1989. Introduction to Quantitative Genetics. 3rd edn. Longman, New York.

Herrnstein, R.J., \& C. Murray, 1994. The Bell Curve: Intelligence and class structure in American life. Free Press, New York.

Hirsch, J., 1963. Behavior genetics and individuality understood: Behaviorism's counterfactual dogma blinded the behavioral sciences to the significance of meiosis. Science 142: 1436-1442.
Hirsch, J., 1967. Behavior-genetic Analysis. In Behavior-Genetic Analysis edited by J. Hirsch, McGraw-Hill, New York.

Kempthorne, O., 1978. Logical, epistemological and statistical aspects of nature-nurture data interpretation. Biometrics 34: 123.

King, J.C., 1981. The Biology of Race. University of California Press, Berkeley.

Lawrence, P.A., 1992. The Making of a Fly: The Genetics of Animal Design. Blackwell Scientific Publications, London.

Lerner, I.M., 1958. The Genetic Basis of Selection. Wiley, New York.

Lewontin, R.C., 1974. The analysis of variance and the analysis of causes. Am. J. Hum. Genet. 26: 400-411.

Lush, J.L., 1937. Animal Breeding Plans. The Collegiate Press, Ames, IA.

Lush, J.L., 1940. Intra-sire correlations or regressions of offspring on dam as a method of estimating heritability of characteristics. Am. Soc. Anim. Prod. Proc. 33: 293-301.

McClearn, G.E., 1962. The inheritance of behavior in Psychology in the Making: Histories of Selected Research Problems edited by L. Postman, Knopf, New York.

McGuire, T.R. \& J. Hirsch, 1977. General intelligence (g) and heritability $(\mathrm{H} 2, \mathrm{~h} 2)$ in The Structuring of Experience edited by I.C. Uzgiris \& F. Weizmann, Plenum, New York.

Neisser, U., G. Boodoo, T.J. Bouchard, A.W. Boykin, N. Brody, S.J. Ceci, D.F. Halpern, J.C. Loehlin, R. Perloff, R.J. Sternberg \& S. Urbina, 1996. Intelligence: Knowns and unknowns. Am. Psycol. 52: 77-101.

Oxford English Dictionary, 2nd edn. [on-line].

Plomin, R., J.C. DeFries \& G.E. McClearn, 1990. Behavioral Genetics: A Primer (2nd edn). Freeman and Co., New York.

Plomin, R., M.J. Owen \& P. McGuffin, 1994. The genetic basis of complex human behaviors. Science 264: 1733-1739.

Tesser, A., 1993. The importance of heritability in psychological research: The case of attitudes. Psych. Rev. 100: 129-142.

Wahlsten, D., 1990. Insensitivity of the analysis of variance to heredity-environment interaction. Behav. Brain Sci 13: 109-161.

Wahlsten, D., 1994a. The intelligence of heritability. Can. Psych. 35: 244-260.

Wahlsten, D., 1994b. Nascent doubts may presage conceptual clarity: Reply to Surby. Can. Psych. 35: 265-267.

Wright, S., 1920. The relative importance of heredity and environment in determining the piebald pattern of guinea pigs. Proc. Nat. Acad. Sci. 6: 320-332. 